\title{
High-content analysis of three-dimensional tumor spheroids: investigating signaling pathways using small hairpin RNA
}

\author{
In the past, creating three-dimensional (3D) tumor spheroids that were suitable for high-throughput \\ screening (HTS) was a difficult and often expensive process. We describe how to couple easy, \\ controllable 3D spheroid formation in Perfecta3D ${ }^{\circledR}$ Hanging Drop Plates with the acumen ${ }^{\circledR} \mathrm{X}^{\mathrm{B}}$ high- \\ content imager for rapid, multicolor, whole-well quantification. This process provides researchers with \\ a highly efficient method to achieve physiologically relevant tumor models in an HTS format.
}

\section{Introduction}

In cancer drug discovery, gene knockdown by RNA interference (RNAi) has demonstrated tremendous success as a research tool for the rapid identification of novel genes and signaling pathway candidates that could make ideal targets. Traditionally, in vitro studies utilizing RNAi have been conducted in cells grown on two-dimensional (2D) surfaces. Such studies fail to take into account the cell-to-cell and cell-to-extracellular matrix interactions that occur in patient tumor biology.

Conducting RNAi screens in 3D, particularly when targeting genes that affect these interactions, may reveal more biologically relevant signaling pathways that occur between complex layers of cells and their surrounding matrix proteins. 3D cell spheroids, which are microscale 3D aggregates of cells, possess characteristics that make them ideal for anticancer drug screening: they are simple to form, have cell-to-cell and cell-to-matrix interactions that more accurately mimic in vivo physiology, and can be utilized with high-content imaging devices for HTS.

Here, we describe the use of 3D spheroids formed in Perfecta3 ${ }^{\circledR}$ Hanging Drop Plates (HDPs) as an HTS colorectal cancer model to test the effects of genetic loss of function on spheroid formation and maintenance. Spheroid formation was analyzed with the acumen ${ }^{\circledR}$ eX3 high-content imaging system.

High-content analysis of 3D spheroid cultures

An HTS 3D spheroid model of colorectal cancer tumor formation was developed to test whether the silencing of specific integrin receptor

Shane R Horman ${ }^{1}$, Jeremy To ${ }^{1}$, Anthony P Orth ${ }^{1}$, Nicky Slawny ${ }^{2}$, Meghan J Cuddihy ${ }^{2} \&$ Diana Caracino ${ }^{3}$

${ }^{1}$ Gene Core, Genomics Institute of the Novartis Research Foundation, San Diego, California, USA. ${ }^{23 D}$ Biomatrix Inc., Ann Arbor, Michigan, USA. ${ }^{3}$ TTP LabTech Inc., Cambridge, Massachusetts, USA. Correspondence should be sent to N.S. (nicky.slawny@3dbiomatrix. com). and receptor tyrosine kinase (RTK) genes inhibited spheroid formation.

$3 \mathrm{D}$ spheroid cultures were generated in the Perfecta3 $\mathrm{D}^{\circledR} \mathrm{HDPs}$ from 3D Biomatrix. Perfecta3 ${ }^{\circledR}$ HDPs are perfectly suited for the experiments described here because they enable easy spheroid formation and are available in a 384-well HTS format (Fig. 1). After formation, spheroids were transferred to assay plates for imaging with the high-content screening instrument acumen ${ }^{\circledR}$ eX3 from TTP LabTech. The acumen ${ }^{\circledR}$ eX3 screening instrument (Fig. 2) performs multicolor, whole-well imaging and is unique in that its wide depth of field is sufficient to capture spheroids without requiring Z-stack assembly. The acumen ${ }^{\circledR}$ eX3 allowed for rapid quantification of spheroid formation.

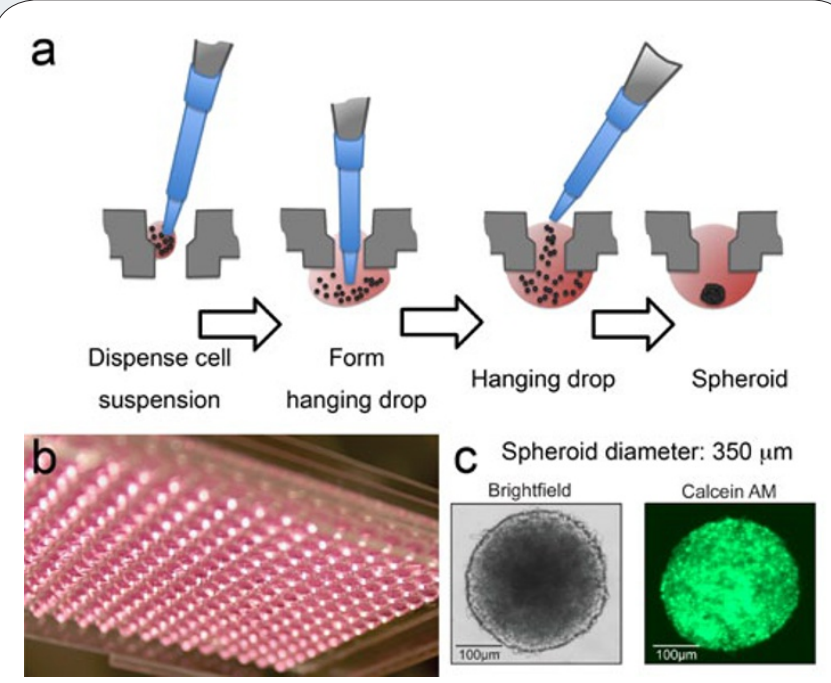

Figure $\mathbf{1}$ | Consistent spheroid formation in high-throughput screening format (a) 384-well Perfecta3D ${ }^{\circledR}$ Hanging Drop Plates (HDPs) facilitate the culture of spheroids within a hanging drop. The user pipettes the cell suspension into each well and the spheroid self-assembles. (b) Drops hang stably below each well. (c) HCT 116 colon cancer spheroids formed in Perfecta3D ${ }^{\circledR}$ HDPs were uniform, with a mean diameter of $350 \mu \mathrm{m}$. 


\section{Establishing small hairpin RNAi infection conditions for the 3D spheroid model}

Prior to initiation of the RNAi screen, HCT 116 colorectal carcinoma cells demonstrated reproducible spheroid formation with a consistent mean diameter of $350 \mu \mathrm{m} \pm 25 \mu \mathrm{m}$ in the 384 - well Perfecta3D ${ }^{\circledR}$ HDPs

(Fig. 1c).

To determine stable gene knockdown that would be suitable for high-content analysis, two methods of viral infection were assessed with colon tumor spheroids. For the EGFP-reporter gene method, 5,000 HCT 116 cells in $25 \mu \mathrm{l}$ of McCoy's 5A medium were dispensed per well into a 384-well Perfecta3D ${ }^{\circledR}$ HDP. Immediately after, $5 \mu \mathrm{l}$ of viral supernatant containing a gene-specific short hairpin RNA (shRNA)-EGFP-lentivirus or a nontargeting shRNA-EGFP-lentivirus control was dispensed into the HDPs. Infection and spheroid formation occurred for $3 \mathrm{~d}$ under standard growth conditions.

For the puromycin (puro)-selection method, 50,000 HCT 116 cells in standard, flat-bottom, 96-well plates were incubated overnight in medium with viral supernatant containing a gene-specific shRNApuro-lentivirus or a nontargeting shRNA-puro-lentivirus control. After $24 \mathrm{~h}$ the transduced cells were selected in $2 \mu \mathrm{g} / \mathrm{ml}$ puromycin for $3 \mathrm{~d}$. Cells were then trypsinized, washed and transferred to the 384-well Perfecta $3 D^{\circledR}$ HDPs $(30 \mu$ l per well), and spheroids were allowed to form for $3 \mathrm{~d}$.

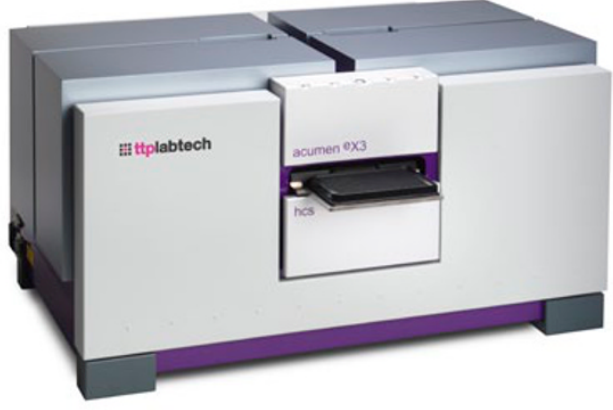

Figure 2 | acumen ${ }^{\oplus}$ eX3 high-content imager for analysis of 3D spheroid formation

\section{High-content analysis of the 3D spheroid model post-} infection

For analysis of viability and spheroid formation after viral infection, the shRNA-EGFP-lentivirus spheroids were transferred into 384-well, black-sided, clear-bottom imaging plates (Greiner) and imaged via the acumen ${ }^{\circledR}$ eX3 high-content imager (Fig. 3a). The nontargeting shRNA-EGFP-lentivirus and the ensuing viral infection did not noticeably alter spheroid formation, indicating that this is an efficient system to deliver shRNAs to spheroids.

\section{Effects of RTK and integrin gene suppression on spheroid formation}

The puro-selection method was utilized to infect cells with shRNA-expressing lentiviruses against platelet-derived growth factor receptor- $\alpha$ (PDGDFR $\alpha), P D G D F R \beta$, integrin $\beta 1$ (ITG $\beta 1)$ or a nontargeting shRNA-puro-lentivirus control (NT control). a
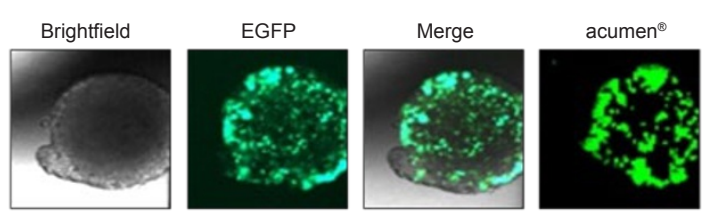

b

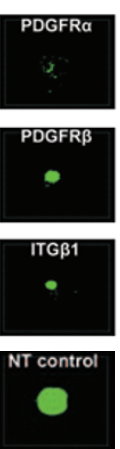

Figure $\mathbf{3}$ | Lentiviral vector shRNA knockdown in colon tumor spheroids and high-throughput screening analysis. (a) A colon tumor spheroid infected with an EGFP-expressing lentivirus and imaged using confocal microscopy and laserscanning imaging (acumen ${ }^{\circledast}$ eX3 instrument). (b) Relative spheroid formation of HCT 116 colon tumor cells after infection with shRNA-expressing lentiviruses against PDGDFRa, PDGFR $\beta$, ITG $\beta 1$ or NT control. Inset images show representative spheroids stained with calcein AM and imaged on the acumen ${ }^{\circledR}{ }^{\circledR} X 3$. Relative spheroid formation was calculated by dividing total calcein AM-positive cytokine-specific shRNA or NT control area by calcein AM-positive NT control area. Errors bars represent standard deviation. ${ }^{* *} P<0.001$

After $3 \mathrm{~d}$, spheroids were transferred into 384-well assay plates as described above, stained with calcein AM and imaged on the acumen $^{\circledR}$ eX3. Relative spheroid formation was calculated by dividing the average total intensity measurement of the shRNA-infected spheroids by the average total intensity measurement of the NT control spheroids. As shown in Figure $\mathbf{3 b}$, genetic knockdown of $P D G D F R \alpha$, $P D G D F R \beta$ or ITG $\beta 1$ led to significantly less spheroid formation than that seen with the NT control.

\section{Conclusion}

Combining RNAi technology with 3D spheroid models and acumen ${ }^{\circledR}$ high-content imaging revealed that lowering PDGDFR $\alpha, P D G F R \beta$ or ITG $\beta 1$ expression significantly reduced spheroid formation consistent with what has been reported in the literature. These results demonstrate that viral genomic reagents can be used in conjunction with $3 \mathrm{D}$ spheroid culture in Perfecta3 $D^{\circledR}$ HDPs and high-content analysis with the acumen ${ }^{\circledR}$ eX3 to screen RNAi libraries in an HTS-compatible format. Growing cells in 3D yields gene expression patterns more similar to those seen in vivo; therefore, interrogating gene function in 3D rather than 2D may significantly accelerate chemotherapeutic drug discovery. This 3D platform may represent a new screening paradigm from which targets related specifically to 3D tumor structural growth can be revealed and exploited for therapeutic intervention.

This article was submitted to Nature Methods by a commercial organization and has not been peer reviewed. Nature Methods takes no responsibility for the accuracy or otherwise of the information provided. 